\title{
Big Rip Singularity in 5D Viscous Cosmology
}

\author{
G.S. Khadekar ${ }^{1, *}$ and N.V. Gharad ${ }^{2}$ \\ ${ }^{I}$ Department of Mathematics, Rashtrasant Tukadoji Maharaj Nagpur University, Mahatma Jyotiba Phule Educational \\ Campus, Amravati Road, Nagpur-440033, India \\ ${ }^{2}$ Department of Physics, Jawaharlal Nehru College, Wadi, Nagpur-440023, India
}

\begin{abstract}
Dark energy of phantom or quintessence nature with an equation of state parameter $\omega$ almost equal to -1 often leads to a finite future singularity. The singularities in the dark energy universe, by assuming bulk viscosity in the framework of Kaluza-Klein theory of gravitation have been discussed. Particularly, it is proved, that the physically natural assumption of letting the bulk viscosity be proportional to the scalar expansion in a spatially 5D FRW universe, can derive the fluid into the phantom region $(\omega<-1)$, even if it lies in the quintessence region $(\omega>-1)$ in the non viscous case. It is also shown that influence of the viscosity term acts to shorten the singularity time but it does not change the nature of singularity in the framework of higher dimensional space time.
\end{abstract}

Keywords: Big Rip, dark energy, future singularity, viscous cosmology.

\section{INTRODUCTION}

A revolutionary development seems to have taken place in cosmology during the last few years. The latest developments of super-string theory and super-gravitational theory have created interest among scientists to consider higherdimensional space-time, for study of the early universe. A number of authors [1-4] have studied physics of the universe in higher-dimensional space-time. Overduin and Wesson [5] have presented an excellent review of higher-dimensional unified theories, in which the cosmological and astrophysical implications of extra-dimension have been discussed.

Kaluza-Klein theory has a long and venerable history. However, the original Kaluza version of this theory suffered from the assumption that the 5-dimensional metric does not depend on the extra coordinate (the cylinder condition). Hence these are proliferation in recent decade of various versions of Kaluza-Klein theory, super gravity and super strings. Kaluza-Klein achievements have shown that 5-dimensional general relativity contains both Einstein's 4-dimensional theory of gravity and Maxwell's theory electromagnetism. In the last decade, a number of authors [6-9] have considered multidimensional cosmological model.

The cosmological observations have provided increasing evidence that our universe is undergoing a late time cosmic acceleration (Perlmutter et al. [10] and Riess et al. [11]). In order to explain the acceleration expansion, cosmologist introduces a new fluid, which posses a negative enough pressure called dark energy. Dark energy model can be divided into two categories: (i) scalar field dark energy models

*Address correspondence to this author at the Department of Mathematics, Rashtrasant Tukadoji Maharaj Nagpur University, Mahatma Jyotiba Phule Educational Campus, Amravati Road, Nagpur-440033, India;

Tel: 91-9011323123; E-mail: gkhadekar@yahoo.com including a quintessence (Wang et al. [12]) and phantom (Caldwell [13]), and (ii) interacting dark energy models, by considering the interaction including Chaplygin gas (Kamenohehik et al [14]), generalized Chaplygin gas (Bento et al. [15]) and a linear equation of state (Babichev et al. [16]). The bulk viscosity in isotopic space bulk and shear viscosity in anisotropic space are presented by (Padmanabham and Chitre [17]). The Planck data show that the Hubble constant $\mathrm{H}$ is the tension with that measure by the several direct probes on $\mathrm{H}$. The Planck data favor $\omega<-1$ when the "supernova legacy survey" is complicated.

The current observations [11, 18-20] strongly suggest that the universe is dominated by a negative pressure component, dubbed dark energy. This component can be characterized by an equation of state parameter $\omega$, which is simply the ratio of the pressure to the density: $\omega=\mathrm{p} / \rho$; for example cosmological constant corresponds to $\omega=1$. While it is often assumed that $\omega \geq-1$ in accordance with weak energy condition, it has long been known [13] that the observations are consistent with $\omega<-1$, which correspond to a dark energy density that increases with time $t$ and the scale factor $a$. If the energy density increases monotonically in the future, then the universe can undergo a future singularity, called the "Big Rip", for which $\rho \rightarrow \infty$ and $a \rightarrow \infty$ at a finite time. In turn, this may lead to the occurrence of negative entropy [21]. One may expect that natural effects, most likely of a quantum mechanical origin, may prevent the Big Rip, as was shown in $[22,23]$.

Viscous cosmological models have been increasingly popular lately. From a hydrodynamics viewpoint, this is quite a natural development, as the inclusion of the viscosity coefficients (there are two of them, shear and bulk) means that one departs physically from the case of an ideal fluid and incorporate the deviations from the thermal equilibrium to first order. The case of an ideal (non-viscous) fluid is, 
after all an idealized model, useful in practice for many situations but not for all, especially not when fluid motion near solid boundaries is involved. In cosmological context, as the cosmic fluid is assumed to be spatially isotropic, the shear viscosity is usually left out. Anisotropic deviations such as those encountered in the Kasner universe are quickly smoothed out being the only coefficient left $\zeta$, the bulk viscosity. Some of the early treatises on viscous cosmology are described [24-28]. A brief review of the singularities in four dimensions and five dimensions in viscous cosmology near future is discussed by [29]. Higher dimensional FRW cosmological model with traversable static wormhole and Big Rip is discussed in detail by Brevik et al. [30]. Recently, Gorbunova and Diego Saez-Gomez [31] described the influence of Casimir effects near the Big Rip singularity in viscous cosmology. Also Padmanabhan and Chitre [17] studied a constant and an oscillating dark energy fluid that crosses the phantom barrier $(\omega<-1)$ and derives the universe evolution to a Big rip singularity.

The present paper is the generalization of the work [32] in the framework of Kaluza-Klein cosmology. The role of bulk viscosity $\varsigma$ in a universe having Big Rip singularity will be studied. For this, it has been considered that Kaluza-Klein cosmology with cosmological constant is equal to zero. The shear viscosity $\varsigma$ will be put equal to zero, in conformity with usual practice. We present the formulation for Kaluza-Klein cosmology when the Equation of State (EoS) is of general form, $\omega=\omega(\rho)$ and examine the consequences of cosmic fluid with bulk viscosity. We discuss some spatial cases. In all the cases, the presence of bulk viscosity tends to promote the big rip phenomenon. Then it is observed that the barrier $(\omega=-1)$ between the quintessence region $(\omega>-1)$ and the phantom region $(\omega<-1)$ can be crossed, as a consequence of the bulk viscosity. The case $\varsigma=\tau \theta$, means that we go a step further; it means that the momentum transfer involves second order quantities in deviation from reversibility, still maintaining the scalar property of $\varsigma$. The general case concerning the dependencies of both the function $f(\rho)$ and the bulk viscosity $\varsigma$ in terms of the density $\rho$ is discussed in detail.

\section{BASIC FORMALISM: KALUZA-KELIN COSMOL- OGY}

We assume the Kaluza-Klein type cosmological model in the form:

$d s^{2}=-d t^{2}+\mathfrak{a}^{2}(t) \sum_{i=1}^{4}\left(d x^{i}\right)^{2}$,

where $\mathfrak{a}(\mathrm{t})$ is a scale factor and the spatial curvature $\mathrm{k}$, as well as cosmological constant is equal to zero. The Hubble parameter $H=\frac{a}{a}$, and scalar expansion $\theta=\bigcup_{; \mu}^{\mu}=4 H, \cup^{\mu}$, with 5 being the velocity of the fluid.

We assume the equation of state (EoS) in the form:

$\mathrm{P}=\omega(\rho) \rho$,

where the thermodynamical variable $\omega(\rho)$ is an arbitrary function of $\rho$. Similarly, we assume the bulk viscosity is arbitrary $\varsigma=\varsigma(\rho)$.
The five dimensional Einstein field equations are:

$$
\begin{aligned}
& \theta^{2}=\frac{64 \pi G}{3} \rho, \\
& \frac{\ddot{a}}{a}+\frac{\theta 2}{16}=-\frac{8 \pi G}{3}(P-\zeta \theta) .
\end{aligned}
$$

The effective pressure $\bar{p}=p-\zeta \theta$ on the thermodynamical grounds, in conventional physics $\varsigma$ has to be positive quantity.

The energy conservation equation leads to:

$\dot{\rho}+(\rho+p) \theta=\zeta \theta^{2}$.

From the above equations, the differential equation for the scalar expansion, with $\omega$ and $\zeta$ as a free parameter can be derived as:

$\dot{\theta}+\frac{1}{2}(1+\omega) \theta^{2}-\frac{32 \pi G}{3} \zeta \theta=0$.

This equation can be written as a differential equation for $\rho$ in the view of the relation:

$\theta=\frac{\sqrt{64 \pi G}}{\sqrt{3 \rho}} \cdot \dot{\rho}$. We will be interested in the region around $\omega=-1$, so it is convenient to introduce the EoS:

$p=-\rho-f(\rho)$.

Then the above Eq. (6) takes the form:

$\dot{\rho}-\sqrt{64 \pi G \rho / 3} f(\rho)-\frac{64 \pi G}{3} \zeta \rho=0$,

we denote the starting value of $\rho$ by $\rho_{0}$. From Eq. (8) we obtain:

$$
\frac{\sqrt{3}}{\sqrt{64 \pi G}} \int_{\rho 0}^{\rho} \frac{d \rho}{f(\rho)\left[\sqrt{\rho}+\frac{\sqrt{64 \pi G}}{\sqrt{3}} \varsigma \frac{\rho}{f(\rho)}\right]} .
$$

This is the general relation between the cosmological time $\mathrm{t}(>0)$ and the density $\rho$.

In the following section, we solve Eq. (9) for different values, a bulk viscosity function $\zeta$.

\section{THE CASE WHEN $\omega=$ CONSTANT}

Let us put

$$
f(\rho)=\alpha \rho,
$$

where $\alpha$ is a constant. This means that $p=-(1+\alpha) \rho$. We investigate the following with some different choice for $\zeta$.

3.1. $\zeta=0$

This is the non viscous case, Eq. (9) gives

$t=\frac{2 \sqrt{3}}{\sqrt{64 \pi G \alpha}}\left(\frac{1}{\sqrt{\rho_{0}}}-\frac{1}{\sqrt{\rho}}\right)$.

If $\alpha>0$ for $t \rightarrow t_{s}$, the Big Rip singularity takes place i.e. $\alpha>0$ a finite value of the time $t$ is compatible with a Big Rip singularity $(\rho=\infty)$. This is the conventional phantom case corresponds to $(\omega<-1)$. 

the form:

By using (11) we can also express density $\rho$ with time in

$$
\rho=\rho_{0}\left(1-\frac{1}{2} \theta_{0} \alpha t\right)^{-2}
$$

where the initial scalar expansion is

$\theta_{0}=\sqrt{\frac{64 \pi G \rho_{0}}{3}}$.

Similarly the scalar expansion is

$$
\theta=\theta_{0}\left(1-\frac{1}{2} \theta_{0} \alpha t\right)^{-1}
$$

and the scale factor $\mathfrak{a}$ is

$$
\mathfrak{a}=\mathfrak{a}_{0}\left(1-\frac{1}{2} \theta_{0} \alpha t\right)^{-\frac{1}{2 \alpha}}
$$

We note here that all the quantities $\rho(\mathrm{t}),(\mathrm{t})$ diverge at Big Rip and it is observed from the expression of the scale factor $\mathfrak{a}$ and the energy density $\rho$ that $\frac{\rho}{\rho_{0}}=\left(\frac{\mathfrak{a}}{a_{0}}\right)^{4 \alpha}$.

\section{2. $5=$ Constant}

In this case (9) takes the form:

$t=\frac{\sqrt{3}}{\alpha \sqrt{64 \pi G}} \int_{\rho_{0}}^{\rho} \frac{d p}{\rho^{\frac{3}{2}}\left[1+\sqrt{\frac{64 \pi G}{3} \alpha \sqrt{\rho}}\right]}$.

Here the integration start from $t_{0}=0$, the present time. The Big Rip is inevitable if this integral is finite for $\rho \rightarrow \infty$. If the fluid is non viscous, $\zeta=0$ then the results of the previous case are recovered. However, for the viscous fluid, the situation is different: $\sqrt{\frac{64 \pi G}{3}} \frac{\varsigma}{\alpha \sqrt{\rho}} \rightarrow 0$ as, $\rho \rightarrow \infty$ the integral reduces effectively to $\int \rho^{-3 / 2} d p \alpha \rho^{-1 / 2}$ for large $\rho$.

Thus, we reach the following important conclusion: if $\varsigma=$ constant , the Big Rip singularity occurs.

The expression for the $\theta$ can be calculated from Eq. (6):

$\theta=\frac{\theta_{0} e^{\frac{t}{t_{c}}}}{1-\frac{1}{2} \alpha t_{c} \theta_{0}\left(e^{\frac{t}{t_{c}}}-1\right)}$,

where

$t_{c}=\left(\frac{32 \pi G \zeta}{3}\right)^{-1}$.

Correspondingly,

$\mathfrak{a}(t)=\mathfrak{a}_{0}\left[1-\frac{1}{2} \alpha t_{c} \theta_{0}\left(e^{\frac{t}{t_{c}}}-1\right)\right]^{-\frac{1}{2 \alpha}}$,

and from Eq. (3):

$\rho=\frac{\rho_{0} e^{t / t c}}{\left[1-\frac{1}{2} \alpha t_{c} \theta_{0}\left(e^{\left.t / t_{c-1}\right)}\right]^{2}\right.}$.

Thus, at the time $\mathrm{t}=t_{s}$, where $t_{s}=t_{c} \ln \left(1+\frac{2}{\alpha t_{c} \theta_{0}}\right)$,

there occurs a Big Rip; all the quantities $(t), \mathfrak{a}(\mathrm{t}), \rho$ diverges.

3.3. $\zeta=\tau \theta$

In this case, we assumed that the constant $\tau$ has to be positive as $\zeta$ is positive. From (3) the bulk viscosity as a function of energy density $\rho$ :

$\zeta(\rho)=\tau \sqrt{64 \pi G \rho / 3}$.

Again from Eq. (9) with the help of Eq. (22) we get:

$t=\frac{6 \sqrt{3}}{\sqrt{64 \pi G}(3 \alpha+64 \pi G \tau)}\left(\frac{1}{\sqrt{\rho_{0}}}-\frac{1}{\sqrt{\rho}}\right)$.

From this condition it follows that the condition for a Big $\operatorname{Rip}(\rho=\infty)$ to occur in a finite time $\mathrm{t}$ is that the prefactor is positive,

$3 \alpha+64 \pi G \tau>0$.

Eq. (24) is the most important result of this paper. Even if we start from the situation where $\omega>-1$ (i.e. $\alpha<0$ ), corresponding to the quintessence region for an ideal fluid, the presence of a sufficiently large bulk viscosity will make the Eq. (24) satisfied and thus derived the fluid into the Big Rip singularity. Somewhat surprisingly, it is the reduction of the thermodynamic pressure generated by the viscosity which in turn causes the barrier $\omega=-1$ to be crossed.

The system is actually most easily analyzed by going back to Eq. (6) for scalar expansion. Introducing the effect in the parameter

$\bar{\alpha}=3 \alpha+64 \pi G \tau$,

we can write the equation as:

$\theta-\frac{1}{6} \alpha \theta^{2}=0$

From this, it follows that $\rho(\mathrm{t}), \theta(\mathrm{t})$ and $\mathfrak{a}(\mathrm{t})$ can actually be found from the non-viscous expression Eq. (12) to Eq. (15), only with replacement $\alpha \rightarrow \bar{\alpha}$. This is the conventional case, corresponding to constant viscosity coefficient. The present case $\zeta=\tau \theta$ means that we go state further; it means that the momentum transfer involves second order quantities in the derivation from reversibility, still maintaining the scalar property of $\zeta$. In this case, the expressions for $(\mathrm{t}), \mathfrak{a}(\mathrm{t})$ and $\rho$ are as follows:

$$
\begin{aligned}
& \theta=\frac{1}{\left(\theta_{0}-t_{d} t\right)}, \\
& \mathfrak{a}(t)=\mathfrak{a}_{0}\left(1-\theta_{0} t_{d} t\right)^{-1 / 4 t_{d},}
\end{aligned}
$$

and

$$
\rho=\frac{\rho_{0}}{\left(1-\theta_{0} t_{d} t\right)^{2}}
$$

where $t_{d}=\left(\frac{3 \alpha+64 \pi G \tau}{6}\right)$. 
Thus, all above quantities diverge at the Big Rip. We note here that

$$
\frac{\rho}{\rho_{0}}=\left(\frac{\mathfrak{a}}{\mathfrak{a}_{0}}\right)^{8 t_{d}} .
$$

\subsection{More General Case}

For the more general case we have considered the function $f(\rho)$ and the bulk viscosity $\zeta$ in terms of the density $\rho$ [14]:

$f(\rho)=\alpha \rho^{\beta+1 / 2}, \zeta=\tau \rho^{\gamma}$

In this case from Eq. (9) we get:

$t=\frac{\sqrt{3}}{\sqrt{64 \pi G}} \int_{\rho_{0}}^{\rho} \frac{d p}{\rho\left[\alpha \rho^{\beta}+b \rho^{\gamma}\right]}$.

This expression can be integrated in terms of hyper geometric function, the result being [15]:

$t=\frac{\beta \rho^{-\gamma}}{\gamma b(\gamma-\beta)}+\frac{\beta \rho^{-\gamma}}{\gamma b(\gamma-\beta)} \times \mathrm{X}(\rho)$,

where $\mathrm{b}=\sqrt{\frac{64 \pi G}{3}} \tau$ and $\mathrm{X}(\rho)$

$=\left[-\beta+(\beta-\gamma)_{2} F_{1}\left(1, \frac{\gamma}{\gamma-\beta}, 1+\frac{\gamma}{\gamma-\beta}, \frac{A}{\beta} \rho^{\beta-\gamma}\right)\right]$.

Some particular cases deserve special attention. When the bulk viscosity is zero (i.e. $\tau=0$ ) and $\beta=\frac{1}{2}$, we again obtain the results discussed in the case (i) and for the bulk viscosity is constant i.e. $\gamma=0$ and $\beta=\frac{1}{2}$, we obtain the results of case (ii) i. e. $\gamma=0$ for $\beta>0$ we have Big Rip.

In the subclasses of case when $\gamma=\beta>0$, one obtains the value $\rho=\infty$ is reached a finite and positive time,

$$
t_{B R}=\frac{\sqrt{3} \rho_{0}-\gamma}{\sqrt{6 \pi G} \gamma(a+b)},
$$

therefore, we returned to the Big Rip situation in all these cases. The case $\zeta=\tau \theta$ has been considered in a cosmological context [16].

\section{CONCLUSION}

In summary, we have considered the role of viscous fluid (or inhomogeneous (imperfect) EoS) in a Big Rip cosmology in the framework of Kaluza-Klein theory of gravitation. In the Big Rip, the scale factor and energy density diverge in the singularity at the finite future time. In the ACDM model, there is no such divergence because the dark energy density remains constant. We have admitted a general form for the bulk viscosity, $\zeta=\zeta(\rho)$ and discussed some spatial cases.

For case (i), i.e. $\zeta=0$ (non-viscous case) we note that $\frac{\rho}{\rho_{0}}=\left(\frac{\mathfrak{a}}{a_{0}}\right)^{4 \alpha}$ and all the quantities $\rho(\mathrm{t}), \theta(\mathrm{t})$ and $\mathfrak{a}(\mathrm{t})$ diverge at the Big Rip. We assume the simple form for the equation of state $p=-(1+\alpha) \rho$, we show in Eqs. (12) to (15) that the viscosity acts so as to shorten the singularity time, but it does not change the nature of the singularity.
For case (ii) i.e. $\zeta=$ constant, it is observed that $\mathfrak{a} \rightarrow \infty, \theta \rightarrow \infty, \rho \rightarrow \infty$ fort $\rightarrow t_{s}$. As $\alpha$ has to be a positive quantity correspondingly to $\omega=-(1+\alpha)<-1$. When $\zeta \rightarrow 0$ the results of case (i) are recovered. The existence of viscosity coefficient in a fluid is due to the thermodynamic irreversibility of the motion.

In case (iii), i.e. $\zeta=\tau \theta$, from Eq. (23), it follows that the condition for a Big Rip $(\rho=\infty)$ to occur in finite time $\mathrm{t}$ is that the prefactor $(3 \alpha+64 \pi G \tau)>0$. For the Kaluza-Klein type FRW universe, it is also shown that universe can derive the fluid into the phantom region $(\omega<-1)$ even if it lies in the quintessence region $(\omega>-1)$ in the non-viscous case. A somewhat stronger influence from cosmology is experienced, as in the case where the viscosity is large enough to make the fluid pass through the phantom barrier $\omega=-1$ into the phantom region (Brevik and Gorbunova [27]).

In general, case we have considered $f(\rho)$ and the bulk viscosity $\zeta$ in terms of density $\rho$. It is observed that when $\gamma=0$ and $\beta=1 / 2$ we again obtained the results of case (ii) and for $\gamma=\beta>0$ we get $\rho \rightarrow \infty$ at the finite time $t_{B R}=\frac{\sqrt{3} \rho_{0}-\gamma}{\sqrt{64 \pi G} \gamma(a+b)}$. Thus, it is shown that Big Rip singularity is allowed in higher dimensional space time.

\section{CONFLICT OF INTEREST}

The authors confirm that this article content has no conflicts of interest.

\section{ACKNOWLEDGEMENTS}

Declared none.

\section{REFERENCES}

[1] Sahdev D. Perfect fluid higher-dimensional cosmologies. Phys Rev D 1984; 30: 2495 .

[2] Emelyanov VM, Nikitin YuP, Rozental JL, Berkov AV. Physics in multidimension spaces and the beginning in meta galaxy. Phys Rep 1986; 143: 1 .

[3] Chatterjee S, Bhui B. Homogeneous cosmological model in higher dimension. Mon Not R Astron Soc 1990; 247: 57.

[4] Chatterjee S, Bhui B. Spherical symmetry and mass-energy in higher dimensions. Int J Theor Phys 1993; 32: 671.

[5] Overduin JM, Wesson PS. Kaluza-Klein gravity. Phys Rep 1987; 283: 303 .

[6] Wesson PS. A physical interpretation of Kaluza-Klein cosmology. Astrophys J 1992; 394: 19.

[7] Chatterjee S, Panigrahi D, Banerjee A. Inhomogeneous KaluzaKlein cosmology. Class Quant Grav 1994; 11: 371.

[8] Chatterjee S, Bhui B, Basu MB, Banerjee A. Inhomogeneous model with a cosmological constant. Phys Rev D 1994; 50: 2924.

[9] Chakraborty S, Roy A. String-dust in higher-dimensional cylindrically symmetric cosmological model. Int J Mod Phys D 1999; 8: 645 .

[10] Perlmutter S. Discovery of a supernova explosion at half the age of the universe. Nature 1998; 391: 51.

[11] Riess AG. Observational evidence from supernovae for an accelerating universe and cosmological constant. Astron J 1998; 116: 1009.

[12] Wang L, Caldwell RR, Ostriker JP, Steinhardt PJ. Cosmic concordance and quintessence. Astrophys J 2000; 530: 17-35. 
[13] Cadwell RR. A phantom menace? Cosmological consequence of dark energy component with super negative equation of state. Phys Lett B 2002; 545: 23.

[14] Kamenshehik A, Moschella U and Pasquier V. An alternative to quintessence. Phys Lett B 2001; 511: 265.

[15] Bento MC, Bertolani O and Sen A. Generalized Chaplygin gas, accelerated expansion and dark energy matter unification. Phys Rev D 2002; 66: 043507.

[16] Babichev E, Doknchaev V, Eroshenko Xu. Dark energy cosmology with generalized linear equation of state. Class Quant Grav 2005; 22: 143 .

[17] Padmanabhan T, Chitre SN. Viscous Universe. Phys Lett A 1987; 120: 433.

[18] Perlmutter S. Measurements of $\omega$ and $\Lambda$ from 42 high-redshift supernovae. Astrophys J 1999; 515: 565.

[19] Kowalskin M, Rubin D, Aldering G, et al. Improved cosmological constants from new, old and combined supernovae dataset. Astrophys J 2008; 886: 749.

[20] Hicken M, Wood-Vasey WM, Blondin S, et al. Improved dark energy constraints from $\approx 100$ New CfA supernovae type Ia light curves. Astrophys J 2009; 700: 1097.

[21] Brevik I, Nojiri S, Odintsov SD, Vanzo I. Entropy and universality of the Cardy-Verlind formula in dark energy. Phys Rev D 2004; 70: 043520 .
[22] Elzalde E, Nojiri S, Odintsov SD. Late-time cosmology in a (phantom) scalar tensor: dark energy and the cosmic sped-up. Phys Rev D 2004; 70: 043539 .

[23] Nojiri S, Odintsov SD. Quantum escape of sudden future singularity. Phys Lett B 2004; 595: 1.

[24] Weinberg S. Gravitation and cosmology. Astrophys J 1971; 168: 175.

[25] Gron O. Viscous inflationary universe model. Astrophys Space Sci 1990; 173: 191.

[26] Brevik I, Heen LT. Remark on the viscous concept in the early universe. Astrophys Space Sci 1994; 219: 99.

[27] Brevik I, Gorbunova O. Dark energy viscous cosmology. Gen Rel Grav 2005; 37: 2039.

[28] Brevik I, Gorbunova O. A brief review of singularities in 4D and 5D viscous cosmologies near the future singularity. arXiv:0811.1129v1 [gr-qc] (2008).

[29] Ahmand Rami EI-Nabulsi. Accelerated higher dimensional cosmology with a traversable static wormhole and a big rip. Gen Rel Grav 2011; 43: 261.

[30] Brevik I, Gorbunova O, Diego Saez-Gomez. Casimir effects near the big rip singularity in viscous cosmology. Gen Rel Grav 2010; 42: 1513 .

[31] Gorbunova O, Diego Saez-Gomez. The oscillating dark energy and cosmological casimir effect. Open Astron J 2010; 3: 73.

[32] Brevik I, Elzalde E, Nojiri S, Odintsov SD. Viscous little rip. Phys Rev D 2011; 84: 103508.

Received: August 31, 2013

(c) Khadekar and Gharad; Licensee Bentham Open.

This is an open access article licensed under the terms of the Creative Commons Attribution Non-Commercial License (http://creativecommons.org/licenses/by-nc/3.0/) which permits unrestricted, non-commercial use, distribution and reproduction in any medium, provided the work is properly cited. 